\title{
Crescimento infantil comparado com as referências NCHS e o padrão WHO/2006
}

\author{
Child growth compared with the NCHS reference \\ and WHO/2006 standard
}

Maria da Graça Mouchrek JALDIN'

Feliciana Santos PINHEIRO ${ }^{1}$

Alcione Miranda dos SANTOS 2

Nivaldo Costa MUNIZ ${ }^{3}$

\section{R E S U M O}

\section{Objetivo}

Este trabalho teve como objetivo analisar o crescimento de crianças em regime de aleitamento materno exclusivo até o sexto mês, bem como comparar seu peso e comprimento com a referência do National Center for Health Statistics 1978 e com o padrão da World Health Organization 2006.

\section{Métodos}

Trata-se de estudo longitudinal realizado em um Banco de Leite Humano, na cidade de São Luís, Maranhão, entre outubro de 2007 e novembro de 2008. Foi feita amostragem não probabilística com 328 crianças nascidas a termo e submetidas a aleitamento materno exclusivo. Finalizaram o estudo 181 crianças. Avaliou-se o crescimento por meio de médias e desvio-padrão, comparando-se os resultados com aqueles dois parâmetros.

\section{Resultados}

Os meninos mostraram-se mais pesados que as meninas, do primeiro ao sexto mês $(p<0,05)$. A maior velocidade no ganho ponderal ocorreu nos dois primeiros meses de vida, em ambos os sexos. Quanto ao peso, as meninas apresentaram peso médio superior ao padrão da World Health Organization 2006, ao nascer e do terceiro ao sexto mês $(p<0,05)$, enquanto os meninos mostraram peso superior do quarto ao sexto mês $(p<0.05)$. Já em relação à referência do National Center for Health Statistics 1978, ambos os sexos apresentaram peso superior desde o nascimento até o sexto mês $(p<0,05)$, exceto os meninos ao nascer. Quanto ao tamanho, as meninas apresentaram comprimento médio semelhante ao padrão da World Health Organization 2006, porém menor do que a outra referência, ao nascer e no sexto mês $(p<0,05)$. Já os meninos obtiveram comprimento médio

\footnotetext{
1 Universidade Federal do Maranhão, Centro de Ciências Biológicas e da Saúde, Departamento de Medicina III. Av. dos Portugueses, s/n., Campus Universitário do Bacanga, 65080-040, São Luís, MA, Brasil. Correspondência para/Correspondence to: MGM JALDIN. E-mail: <gracajaldin@yahoo.com.br >.

2 Universidade Federal do Maranhão, Centro de Ciências Biológicas e da Saúde, Departamento de Saúde Pública. São Luís, MA, Brasil.

${ }^{3}$ Universidade Federal do Maranhão, Centro de Ciências Exatas e Tecnologia, Departamento de Matemática. São Luís, MA, Brasil.
} 
$18 \mid$ MGM JALDIN et al.

inferior, tanto quando comparados ao padrão da World Health Organization 2006, do nascimento ao sexto mês $(p<0,05)$, exceto o quinto; como quando comparados à referência da National Center for Health Statistics 1978, ao nascer e no primeiro, no quarto e no sexto meses de vida $(p<0,05)$.

\section{Conclusão}

O crescimento ponderal das crianças em aleitamento materno exclusivo assemelhou-se mais ao padrão World Health Organization 2006 que à referência National Center for Health Statistics 1978, ao passo que o crescimento linear mostrou-se compatível com os dois parâmetros.

Termos de indexação: Aleitamento materno. Antropometria. Crescimento.

\section{A B S T R A C T}

\section{Objective}

This study assessed the growth of exclusively breastfed infants until six months of age and compared their weight and length with those of the National Center for Health Statistics 1978 reference, and the World Health Organization standard 2006.

\section{Methods}

This was a longitudinal research done at the Human Milk Bank of São Luis, Maranhão, from October 2007 to November 2008. The non-probabilistic sample consisted of 328 exclusively breastfed, full-term infants. A total of 181 infants concluded the study. Growth was assessed by means and standard deviation and the results were compared with those of the National Center for Health Statistics 1978 and World Health Organization 2006.

\section{Results}

Males weighed more than females from the first to the sixth month $(p<0.05)$. The greatest weight gain occurred in the first two months of life for both sexes. The mean female weight was higher than that of the World Health Organization 2006 at birth and from the third to the sixth month $(p<0.05)$; meanwhile, males were heavier from the fourth to the sixth month $(p<0.05)$, but their weights were higher than those of the National Center for Health Statistics 1978, for both genders, from birth to the sixth month ( $p<0.05)$, except for males at birth. The mean male lengths were lower than those of the World Health Organization 2006 from birth to the sixth month $(p<0.05)$, except for the fifth month; they were also lower than the National Center for Health Statistics 1978 at birth and in the first, fourth and sixth months of life $(p<0.05)$; female heights were similar to those of the World Health Organization 2006 and lower than those of the National Center for Health Statistics 1978 at birth and at the sixth month $(p<0.05)$.

\section{Conclusion}

The weight gain of exclusively breastfed children was more similar to that of the World Health Organization 2006 standard than that of the National Center for Health Statistics 1978 reference. Linear growth was appropriate, similar to both the standard and the reference.

Indexing terms: Breast feeding. Anthropometry. Growth.

\section{N T R O D U ÇÃ O}

O alimento completo, que entre inúmeras outras vantagens oferece ao lactente uma nutrição de excelente qualidade, é o leite materno'. São diversos os estudos que ressaltam a importância do Aleitamento Materno Exclusivo (AME) para o estado nutricional infantil até o sexto mês de vida ${ }^{1,2}$. Ressalte-se que o primeiro semestre de vida é o período de mais rápido crescimento humano ${ }^{3}$.
A World Health Organization (WHO) recomenda o aleitamento exclusivo nos seis primeiros meses de vida como único alimento ideal para o crescimento e desenvolvimento infantil. Um dos métodos de avaliação do crescimento é a antropometria, utilizada com a finalidade de monitorar o crescimento, individualmente ou em estudos epidemiológicos ${ }^{4}$. Nesse processo são feitas a pesagem e medição, comparando-se essas medidas a um padrão antropométrico de referência por meio de curvas de crescimento. 
As curvas de crescimento até há pouco tempo recomendadas para uso internacional pela WHO eram as do National Center for Health Statistics (NCHS), reconhecidas como referência NCHSMHO desde 1978, após terem sido normalizadas ${ }^{5}$.

Todavia, diversos pesquisadores, ao avaliarem o crescimento de crianças em AME até seis meses, evidenciaram peso superior ao da curva do percentil 50 da NCHS/19782,6. Outros pesquisadores, com base na mesma referência, mostraram crescimento desacelerado de crianças em AME a partir do terceiro e quarto mês, principalmente com relação ao peso 7,8. A magnitude dos desvios negativos motivou discussões sobre o valor nutricional do leite materno para a promoção de um crescimento sadio, favorecendo o desmame precoce ${ }^{9}$. Com o decorrer do tempo, as curvas da NCHS foram consideradas inadequadas para crianças amamentadas, devido à constatação de uma série de limitações. Dentre as principais, citam-se o fato de as crianças serem alimentadas predominantemente com fórmulas infantis, bem como a situação de as medidas antropométricas serem aferidas ao nascer, no primeiro mês e, depois, a cada três meses ${ }^{10}$.

Entre 1997 e 2003, a WHO realizou um estudo multicêntrico de referência de crescimento, com o objetivo de elaborar novas curvas para avaliação do crescimento e desenvolvimento de crianças de zero a cinco anos, em todo o mundo. Entre outras características, o estudo foi baseado em bebês saudáveis, em aleitamento exclusivo ou predominante, e com mães não fumantes ${ }^{4}$. Embora o padrão WHO tenha sido construído a partir de crianças amamentadas, ele foi proposto para avaliar o crescimento de crianças com qualquer tipo de alimentação ${ }^{11}$.

Dentro desse contexto, o objetivo do presente estudo foi analisar o crescimento de crianças em AME até os seis meses de vida, comparando os dados obtidos com aqueles propostos na referência NCHS/1978 e no padrão WHO/2006.

\section{MÉ T O D O S}

Este trabalho se baseia em um estudo longitudinal realizado no Banco de Leite Humano (BLH) do Hospital Universitário Materno Infantil (HUMI) de São Luís (MA), no período de outubro de 2007 a novembro de 2008. O BLH, considerado um centro de referência em pesquisa sobre aleitamento materno no estado, desde 2004 está inserido no HUMI. É reconhecido como Hospital Amigo da Criança desde 1998, e tem por finalidade assegurar o cumprimento dos dez passos para o sucesso da amamentação.

Foram adotados como critério de inclusão: criança nascida a termo; parto único; Apgar $\geq 7$ no quinto minuto; peso ao nascer entre $2,5 \mathrm{~kg}$ e 4,0kg; em AME desde o nascimento; nascida no HUMI e mantida em alojamento conjunto até a alta hospitalar. Considerou-se AME a definição da WHO/1991. Incluíram-se 27 crianças que fizeram uso ocasional de água ou chá para cólica somente no primeiro mês de vida. Os filhos de mães fumantes e daquelas impossibilitadas de fornecer amamentação exclusiva nos seis primeiros meses não foram incluídos no estudo.

Um total de 328 crianças foi incluído por meio de amostragem não probabilística, no período de outubro de 2007 a junho de 2008, na primeira consulta, com um mês de vida. Nessa ocasião, foram identificadas as crianças e mães que preenchiam os critérios da pesquisa, sendo estas esclarecidas sobre a importância da mesma e sobre os critérios para a inclusão. As mães que aceitaram participar responderam a um questionário sobre identificação, condições sociodemográficas, antecedentes obstétricos, amamentação e dados da criança.

Para aferição do peso e comprimento das crianças, do primeiro ao sexto mês, foram recrutadas duas técnicas de enfermagem do BLH, capacitadas para essa atividade, além da pesquisadora. A equipe foi treinada mediante a realização de um estudo piloto com 30 crianças.

A antropometria realizou-se por meio de técnicas padronizadas e checagem periódica entre 
as profissionais envolvidas. As medidas ao nascer foram colhidas do cartão da criança ou da folha de Declaração de Nascidos-Vivos. As crianças foram pesadas sem roupas, em balança digital (Filizola Ind. Ltda) tipo pesa-bebê, com capacidade máxima de $15 \mathrm{~kg}$ e precisão de $5 \mathrm{~g}$, aferida pelo Instituto Nacional de Metrologia, Normalização e Qualidade Industrial, no início do estudo. A leitura foi realizada em quilogramas, e a balança tarada antes de cada pesagem e checada regularmente para ajuste zero. O comprimento foi aferido com a criança em decúbito dorsal sobre um antropômetro horizontal de madeira (padrão) construído localmente, com subdivisões em milímetros e apoiado em superfície plana, firme e lisa ${ }^{6,12}$.

O ganho ponderal diário foi calculado pela diferença entre o peso da consulta atual e o da consulta anterior, dividida pelo número exato de dias entre as duas consultas ${ }^{13}$. O acompanhamento das crianças ocorreu mensalmente, do primeiro ao sexto mês de vida. Para minimizar a influência da variabilidade da idade sobre os resultados, o intervalo permitido entre a data de aniversário e o dia da consulta nos primeiros quatro meses foi de \pm 1 semana ${ }^{12,14}$, e a partir do quinto mês foi de \pm 2 semanas $^{14}$. $O$ atendimento às crianças foi individual para verificação das medidas antropométricas, observação da mamada e avaliação clínica com o pediatra.

O estudo foi precedido por palestra de incentivo e apoio à prática do $\mathrm{AME}$, com informações sobre amamentação e orientações quanto à técnica correta de amamentar. A presença de familiares foi permitida e incentivada.

Em cada atendimento, perguntou-se às mães sobre a alimentação oferecida à criança, número de mamadas em 24 horas, dificuldades com a amamentação, problemas de saúde e situação vacinal. Para confirmação do AME, utilizaram-se as informações maternas e a observação da mamada. As consultas subsequentes foram agendadas previamente, mas as mães poderiam retornar antes, se as crianças apresentassem intercorrências clínicas. Aos seis meses de idade, as crianças receberam alta do programa, e as mães foram orientadas sobre alimentação complementar e a conveniência de manter o aleitamento materno por, no mínimo, até os dois anos de idade.

Foram excluídas as crianças que não compareceram às consultas mensais no intervalo permitido, bem como as que fizeram uso de outro alimento além do leite humano antes do sexto mês.

O estudo foi aprovado pelo Comitê de Ética em Pesquisa do Hospital Universitário Materno Infantil, Processo n 33104-1076/07, em $25 / 1 / 2008$. Todas as mães assinaram um Termo de Consentimento Livre e Esclarecido antes da inclusão das crianças na amostra.

As medidas antropométricas foram expressas em média, desvio-padrão e analisadas no Stata 9.0, a partir do banco de dados armazenado no Epi Info 2006, versão 3.3.2. Utilizou-se o teste Shapiro Wilk para avaliar a normalidade das variáveis quantitativas e o teste $t$ de Student para comparação das médias com um nível de significância de 5\%.

\section{RESULTADOS}

Foram avaliadas 328 crianças em AME. Entretanto, apenas 181 delas permaneceram até o término do programa, no sexto mês de vida, resultando em 95 meninas (52,50\%) e 86 meninos $(47,50 \%)$. Das outras 147 crianças $(44,82 \%)$, foram excluídas 49 (33,33\%) por não terem comparecido às consultas no intervalo de tempo estipulado e 24 (16,33\%) devido à introdução de outro alimento, enquanto 74 (50,34\%) abandonaram o estudo.

A Tabela 1 mostra diferença estatística com relação ao peso médio entre os sexos, exceto ao nascimento. Meninos duplicaram o peso de nascimento por volta do terceiro mês, e meninas, por volta do quarto mês. O ganho de peso nos seis meses de vida foi de $4,9 \mathrm{~kg}$, nos meninos e $4,4 \mathrm{~kg}$ nas meninas (dados não apresentados em tabela). 
Tabela 1. Peso médio de 181 crianças em aleitamento materno exclusivo, segundo o sexo e a idade. São Luís (MA), 2007-2008.

\begin{tabular}{|c|c|c|c|c|c|}
\hline \multirow{3}{*}{ Idade } & \multicolumn{4}{|c|}{ Peso (kg) } & \multirow{3}{*}{$p$} \\
\hline & \multicolumn{2}{|c|}{ Meninos $(n=86)$} & \multicolumn{2}{|c|}{ Meninas $(n=86)$} & \\
\hline & $M$ & DP & M & DP & \\
\hline Ao nascer & 3,3 & $\pm 0,3$ & $3,3=$ & $=0,3$ & 0,999 \\
\hline $1^{\circ}$ mês & 4,5 & $\pm 0,5$ & $4,2=$ & $=0,5$ & 0,001 \\
\hline $2^{\circ}$ mês & 5,6 & $\pm 0,5$ & $5,2=$ & $=0,5$ & 0,001 \\
\hline $3^{\circ}$ mês & 6,5 & $\pm 0,6$ & $6,0=$ & $=0,6$ & 0,001 \\
\hline $4^{\circ}$ mês & 7,2 & $\pm 0,7$ & $6,7=$ & $=0,7$ & 0,001 \\
\hline $5^{\circ}$ mês & 7,8 & $\pm 0,8$ & $7,2=$ & $=0,9$ & 0,001 \\
\hline $6^{\circ}$ mês & 8,2 & $\pm 0,9$ & $7,7=$ & $=0,9$ & 0,001 \\
\hline
\end{tabular}

M: Média; DP: Desvio-Padrão.

Tabela 2. Ganho ponderal (g) médio diário de 181 crianças em aleitamento materno exclusivo, segundo o sexo e a idade. São Luís (MA), 2007-2008.

\begin{tabular}{|c|c|c|c|c|c|}
\hline \multirow{3}{*}{ Idade } & \multicolumn{4}{|c|}{ Ganho ponderal médio (g/dia) } & \multirow{3}{*}{$p$} \\
\hline & \multicolumn{2}{|c|}{ Meninos $(n=86)$} & \multicolumn{2}{|c|}{ Meninas $(n=86)$} & \\
\hline & M & DP & M & DP & \\
\hline $0-1^{\circ}$ mês & 36,9 & $\pm 10,1$ & 29,6 & $\pm 10,4$ & $<0,001$ \\
\hline $1^{\circ}-2^{\circ}$ mês & 39,7 & $\pm 8,1$ & 33,1 & $\pm 8,4$ & $<0,001$ \\
\hline $2^{\circ}-3^{\circ}$ mês & 29,0 & $\pm 7,6$ & 26,3 & $\pm 9,5$ & 0,017 \\
\hline $3^{\circ}-4^{\circ}$ mês & 23,3 & $\pm 7,4$ & 22,1 & $\pm 7,9$ & 0,162 \\
\hline $4^{\circ}-5^{\circ}$ mês & 19,0 & $\pm 5,8$ & 18,0 & $\pm 7,7$ & 0,155 \\
\hline $5^{\circ}-6^{\circ}$ mês & 14,0 & $\pm 8,5$ & 15,0 & $\pm 7,2$ & 0,184 \\
\hline
\end{tabular}

M: Média; DP: Desvio-Padrão.
Na Tabela 2, verifica-se que o ganho ponderal médio foi maior nos meninos no primeiro trimestre. Em ambos os sexos houve um crescimento acelerado até o segundo mês e, após, uma desaceleração gradual até o sexto mês, mais intensa entre o segundo e o terceiro mês.

Na Tabela 3, observa-se que, nos meninos, o peso médio do quarto ao sexto mês foi superior ao do $\mathrm{WHO} / 2006(p<0,05)$. Nas meninas, foi superior ao nascer e do terceiro ao sexto mês $(p<0,05)$. Nos meninos, do primeiro ao sexto mês, os pesos médios foram superiores ao da NCHS/ 1978, com diferença significante, enquanto nas meninas, desde o nascimento.

Na Tabela 4, verifica-se que o comprimento médio dos meninos foi inferior ao do WHO/2006, do nascimento ao sexto mês, apresentando uma diferença estatística significante, exceto no quinto mês. Nas meninas, o comprimento médio foi menor que o do padrão em todo o primeiro semestre, mas sem significância estatística. Nos meninos, apenas no segundo, terceiro e quinto meses os comprimentos não diferiram dos estabelecidos pela NCHS/1978. Porém, nas meninas não se observou diferença nesses valores do primeiro ao quinto mês.

Tabela 3. Comparação do peso médio $(\mathrm{kg})$ de crianças em aleitamento materno exclusivo com o padrão WHO/2006 e com a referência NCHS/1978, segundo o sexo e a idade. São Luís (MA), 2007-2008.

\begin{tabular}{|c|c|c|c|c|c|c|c|}
\hline \multirow{2}{*}{ Peso (kg) } & \multicolumn{2}{|c|}{ Estudo } & \multirow{2}{*}{ WHO/2006 } & \multirow{2}{*}{$p$} & Estudo & \multirow{2}{*}{ NCHS/1978 } & \multirow{2}{*}{$p$} \\
\hline & $M$ & $\mathrm{DP}$ & & & $M \quad D P$ & & \\
\hline \multicolumn{8}{|c|}{ Meninos $(n=86)$} \\
\hline Ao nascer & $3,3 \pm$ & 0,3 & 3,3 & 1,000 & $3,3 \pm 0,3$ & 3,3 & 0,999 \\
\hline $1^{\circ}$ mês & $4,5 \pm$ & 0,5 & 4,5 & 1,000 & $4,5 \pm 0,5$ & 4,3 & 0,004 \\
\hline $2^{\circ}$ mês & $5,6 \pm$ & 0,5 & 5,6 & 1,000 & $5,6 \pm 0,5$ & 5,2 & $<0,001$ \\
\hline $3^{\circ}$ mês & $6,5 \pm$ & 0,6 & 6,4 & 0,126 & $6,5 \pm 0,6$ & 6,0 & $<0,001$ \\
\hline $4^{\circ}$ mês & $7,2 \pm$ & 0,7 & 7,0 & 0,009 & $7,2 \pm 0,7$ & 6,7 & $<0,001$ \\
\hline $5^{\circ}$ mês & $7,8 \pm$ & 0,8 & 7,5 & $<0,001$ & $7,8 \pm 0,8$ & 7,3 & $<0,001$ \\
\hline $6^{\circ}$ mês & $8,2 \pm$ & 0,9 & 7,9 & 0,003 & $8,2 \pm 0,9$ & 7,8 & $<0,001$ \\
\hline \multicolumn{8}{|c|}{ Meninas $(n=95)$} \\
\hline Ao nascer & $3,3 \pm$ & 0,3 & 3,2 & 0,002 & $3,3 \pm 0,3$ & 3,2 & 0,001 \\
\hline $1^{\circ}$ mês & $4,2 \pm$ & 0,5 & 4,2 & 1,000 & $4,2 \pm 0,5$ & 4,0 & $<0,001$ \\
\hline $2^{\circ}$ mês & $5,2 \pm$ & 0,5 & 5,1 & 0,054 & $5,2 \pm 0,5$ & 4,7 & $<0,001$ \\
\hline $3^{\circ}$ mês & $6,0 \pm$ & 0,6 & 5,8 & 0,002 & $6,0 \pm 0,6$ & 5,4 & $<0,001$ \\
\hline $4^{\circ}$ mês & $6,7 \pm$ & 0,7 & 6,4 & $<0,001$ & $6,7 \pm 0,7$ & 6,0 & $<0,001$ \\
\hline $5^{\circ}$ mês & $7,2 \pm$ & 0,9 & 6,9 & 0,002 & $7,2 \pm 0,9$ & 6,7 & $<0,001$ \\
\hline $6^{\circ}$ mês & $7,7 \pm$ & 0,9 & 7,3 & $<0,001$ & $7,7 \pm 0,9$ & 7,2 & $<0,001$ \\
\hline
\end{tabular}

M: Média; DP: Desvio-Padrão; WHO: World Health Organization; NCHS: National Center for Health Statistics. 
Tabela 4. Comparação do comprimento médio (cm) de crianças em aleitamento exclusivo com o padrão WHO/2006 e com a referência NCHS/1978, por sexo e idade. São Luís (MA), 2007-2008.

\begin{tabular}{|c|c|c|c|c|c|c|c|c|}
\hline \multirow{2}{*}{ Peso (kg) } & \multicolumn{2}{|c|}{ Estudo } & \multirow{2}{*}{ WHO/2000 } & \multirow{2}{*}{$p$} & \multicolumn{2}{|c|}{ Estudo } & \multirow{2}{*}{ NCHS/1978 } & \multirow{2}{*}{$p$} \\
\hline & $\mathrm{M}$ & $\mathrm{DP}$ & & & $\mathrm{M}$ & $\mathrm{DP}$ & & \\
\hline \multicolumn{9}{|c|}{ Meninos $(n=86)$} \\
\hline Ao nascer & 49,1 & $\pm 1,9$ & 49,9 & $<0,001$ & 49,1 & $\pm 1,9$ & 50,5 & $<0,001$ \\
\hline $1^{\circ}$ mês & 54,0 & $\pm 1,7$ & 54,7 & $<0,001$ & 54,0 & $\pm 1,7$ & 54,6 & 0,008 \\
\hline $2^{\circ}$ mês & 57,9 & $\pm 1,8$ & 58,4 & 0,012 & 57,9 & $\pm 1,8$ & 58,1 & 0,303 \\
\hline $3^{\circ}$ mês & 60,9 & $\pm 1,7$ & 61,4 & 0,008 & 60,9 & $\pm 1,7$ & 61,1 & 0,272 \\
\hline $4^{\circ}$ mês & 63,3 & $\pm 1,7$ & 63,9 & 0,002 & 63,3 & $\pm 1,7$ & 63,7 & 0,032 \\
\hline $5^{\circ}$ mês & 65,5 & $\pm 1,9$ & 65,9 & 0,050 & 65,5 & $\pm 1,9$ & 65,9 & 0,054 \\
\hline $6^{\circ}$ mês & 67,0 & $\pm 1,9$ & 67,6 & 0,040 & 67,0 & $\pm 1,9$ & 67,8 & $<0,001$ \\
\hline \multicolumn{9}{|c|}{ Meninas $(n=95)$} \\
\hline Ao nascer & 48,9 & $\pm 2,0$ & 49,1 & 0,342 & 48,9 & $\pm 2,0$ & 49,9 & $<0,001$ \\
\hline $1^{\circ}$ mês & 53,4 & $\pm 1,6$ & 53,7 & 0,070 & 53,4 & $\pm 1,6$ & 53,5 & 0,539 \\
\hline $2^{\circ}$ mês & 56,8 & $\pm 1,8$ & 57,1 & 0,110 & 56,8 & $\pm 1,8$ & 56,8 & 0,999 \\
\hline $3^{\circ}$ mês & 59,5 & $\pm 2,2$ & 59,8 & 0,206 & 59,5 & $\pm 2,2$ & 59,5 & 0,999 \\
\hline $4^{\circ}$ mês & 62,0 & $\pm 1,9$ & 62,1 & 0,608 & 62,0 & $\pm 1,9$ & 62,0 & 0,999 \\
\hline $5^{\circ}$ mês & 63,8 & $\pm 2,1$ & 64,0 & 0,360 & 63,8 & $\pm 2,1$ & 64,1 & 0,166 \\
\hline $6^{\circ}$ mês & 65,4 & $\pm 2,2$ & 65,7 & 0,185 & 65,4 & $\pm 2,2$ & 65,9 & 0,028 \\
\hline
\end{tabular}

M: Média; DP: Desvio-Padrão; WHO: World Health Organization; NCHS: National Center for Health Statistics.

\section{I S CUS S Ã O}

No presente estudo, analisou-se o crescimento de crianças em AME do nascimento aos seis meses de vida. O crescimento dessas crianças foi comparado ao padrão internacional WHO/ 2006 e à referência internacional NCHS/1978.

Entre os sexos, as médias de peso foram iguais às do nascimento, porém do primeiro ao sexto mês, meninos foram mais pesados. Esse mesmo achado foi observado por Donma \& Donma $^{15}$, em estudo sobre alimentação infantil e crescimento no primeiro semestre de vida, na Turquia. Todavia, Dewey et al. ${ }^{16}$, comparando padrão de crescimento de crianças amamentadas e alimentadas por fórmulas, de 0 a 18 meses, mostraram que a média do peso nos meninos em aleitamento materno foi maior que nas meninas, desde o nascimento. Esse fato também foi verificado por Yoneyama et al. ${ }^{17}$ nas crianças em AME, avaliadas ao nascer, no primeiro, terceiro e sexto meses, em estudo feito no Japão, relacionando crescimento ao tipo de alimentação na infância (leite materno e artificial). Marques et al. ${ }^{1}$ encontraram resultado diferente em pesquisa sobre crescimento de 102 crianças amamentadas, ao nascer, no primeiro, quarto e sexto meses, em Belém, onde o peso médio foi maior nas meninas ao nascer, igualou-se ao dos meninos no primeiro mês e foi menor a partir do quarto mês.

Os meninos mostraram um ganho ponderal médio diário superior ao das meninas, principalmente no primeiro trimestre, exceto do quinto ao sexto mês. Simoes \& Pereira ${ }^{13}$ também verificaram diferença em favor dos meninos quanto ao ganho de peso diário. Em ambos os sexos, o período de declínio iniciou-se no segundo mês, estendendo-se até o final do segundo trimestre. Marques et al. ${ }^{1}$ observaram uma redução em torno de $50 \%$ no ganho de peso diário, após o quarto mês, em ambos os sexos. No presente estudo, isso ocorreu após o quinto entre os meninos, e após o sexto mês entre as meninas.

Apesar de as crianças em estudo não terem alcançado, no quinto e sexto meses, os valores de $20 \mathrm{~g} / \mathrm{dia}$ a $25 \mathrm{~g} / \mathrm{dia}$, referidos na literatura como adequados para o segundo trimestre ${ }^{18}$, do ponto de vista clínico elas chegaram ao sexto mês saudáveis e com crescimento satisfatório. De acordo com Augusto \& Souza ${ }^{19}$, a redução que acontece no segundo trimestre ocorre indepen- 
dentemente da duração da amamentação exclusiva, evidenciando um comportamento normal do crescimento.

Os maiores ganhos de peso nos dois primeiros meses de vida e a subsequente desaceleração até os seis meses, notadas neste estudo, foram similares aos mostrados por Otaigbe et al. ${ }^{6}$, Dewey et al. ${ }^{16}$ e Jaimovich et al. ${ }^{20}$. Nesses estudos, as crianças também chegaram aos seis meses de vida com crescimento adequado e satisfatório, apesar da desaceleração.

A duplicação do peso médio de nascimento nos meninos e meninas, que ocorreu por volta do terceiro e quarto mês respectivamente, foi tardia quando comparada àquela registrada no estudo de Yoneyama et al. ${ }^{17}$, segundo o qual os meninos dobraram o peso de nascimento $(3,153 \mathrm{~kg})$ entre o segundo e o terceiro mês, enquanto as meninas $(3,106 \mathrm{~kg})$ o fizeram em torno do terceiro mês. Por outro lado, foi mais precoce que em estudos que evidenciaram peso de nascimento mais elevado, como o de Dewey et al. ${ }^{16}$ $(3,800 \mathrm{~kg}$ vs $3,584 \mathrm{~kg}$, em meninos e meninas, respectivamente), em que a duplicação ocorreu em torno do quinto mês nos meninos, e entre o quinto e sexto nas meninas. Fato semelhante pode ser observado no estudo realizado por Donma \& Donma ${ }^{15}$, no qual o peso médio dos meninos ao nascer $(3,420 \mathrm{~kg})$ dobrou no quarto mês, e nas meninas $(3,420 \mathrm{~kg})$, entre o quarto e o quinto.

Há relatos mostrando que, quanto menor o peso de nascimento, maior a velocidade de ganho ponderal diário ${ }^{13,19}$ e, consequentemente, mais precoce a duplicação ${ }^{21}$. Provavelmente isso ocorre na tentativa de recuperar alguma limitação ocorrida no potencial de crescimento intraútero ${ }^{19}$. Para Augusto \& Souza ${ }^{19}$, a maior velocidade de incremento diário de peso no primeiro trimestre, de modo geral, está relacionada não somente ao menor peso ao nascer, como ao sexo masculino. Pode-se constatar que, neste estudo e nos demais antes referidos, a duplicação de peso ocorreu de modo mais precoce nos meninos.

Esse comportamento pode ser explicado por diferenças anatômicas e fisiológicas entre os sexos, visto que os meninos apresentam crânio e face grandes e, proporcionalmente, maior quantidade de tecido muscular ${ }^{21}$.

Outros autores têm procurado dar explicações sobre a diferença de peso entre os sexos, favorecendo os meninos. Dentre as alegações, incluem-se menor peso das meninas ao nascer e menor quantidade de leite ingerido por elas durante as sucções ${ }^{6,13}$. Apesar de se ter notado, neste estudo, um desempenho melhor nos meninos, fica descartada a influência do peso ao nascer, tendo em vista que ambos os sexos nasceram com pesos iguais e adequados. A hipótese de menor volume de leite ingerido, devido à sucção menos vigorosa, necessita de confirmação científica.

Com base nesses achados, pode-se depreender que a amamentação exclusiva promove o crescimento ponderal infantil do nascimento ao sexto mês, principalmente no primeiro trimestre. Outros estudos relatam que o crescimento adequado só é garantido até o quarto mês de vida ${ }^{22,23}$.

De acordo com Sekiyama et al. ${ }^{24}$, o rápido crescimento nos dois primeiros meses de vida de crianças amamentadas é atribuído à grande ingestão de leite materno ou a seu alto valor nutritivo. Acredita-se que o bom desempenho do crescimento das crianças do presente estudo seja também resultado de orientações sistemáticas e do incentivo que as mães receberam após o parto em Hospital Amigo da Criança e, também, no $B L H$, durante o seguimento de puericultura, com o caráter cíclico das consultas nos primeiros seis meses. Isso permitiu uma abordagem adequada a respeito da importância de mamadas frequentes, sob livre demanda, e a respeito da técnica correta de amamentar e do esvaziamento completo das mamas em cada mamada. Também colaborou o esclarecimento de que o leite do final das mamadas é mais rico em gorduras e de alto teor energético, sendo responsável pela saciedade e maior ganho de peso. Além disso, o apoio constante às mães durante todo o seguimento foi fundamental para o fortalecimento da autoestima, fazendo-as acreditar em sua capacidade de amamentar. A literatura aponta outros trabalhos 
sobre crescimento bem sucedido de crianças amamentadas, como resultado do apoio, incentivo e orientação às mães nutrizes ${ }^{1,6}$.

Quanto ao ganho de peso mais lento, que ocorreu após o segundo mês nas crianças do estudo, esse é provavelmente um fenômeno fisiológico normal, também assinalado por Otaigbe et al. ${ }^{6}$ e por Dewey et $a l .{ }^{16}$, não devendo ser, equivocadamente, considerado como falha no crescimento, pois isso levaria à introdução precoce de alimentos complementares em crianças saudáveis e com bom ritmo de crescimento ${ }^{12}$.

Ao se compararem os resultados deste estudo com o padrão WHO/2006, observa-se que os meninos da amostra obtiveram peso superior, do quarto ao sexto mês $(p<0,05)$, enquanto nas meninas esse fato ocorreu ao nascer e do terceiro ao sexto mês $(p<0,05)$.

As recomendações alimentares da $\mathrm{WHO}$, no estudo multicêntrico, foram: AME ou AMP (aleitamento materno predominante) pelo menos até o quarto mês de vida; alimentos complementares entre o quarto e o sexto mês; e manutenção do aleitamento materno parcial pelo menos até os 12 meses. É importante ressaltar que 99,5\% das crianças do referido estudo iniciaram complementos em torno do sexto mês e que crianças em AMP receberam leite humano como principal fonte de nutrição, além de água e outros líquidos $^{25}$. Chás e sucos, entre outros, são de pouco valor energético. Além do mais, há relato na literatura de que em torno do terceiro e do quarto mês de vida ocorre aumento da necessidade energética diária da criança, devido ao incremento da atividade física e maior tempo em vigília26. Waterlow \& Tomson ${ }^{22}$ estimam que as necessidades energéticas aumentem em torno de 12,0\% após o quarto mês.

O leite materno exclusivo contém todos os nutrientes necessários para atender às demandas de crescimento da criança até os seis meses de idade ${ }^{27}$, ou até em idades mais avançadas ${ }^{28}$. Possivelmente, o fato de as crianças estudadas terem sido exclusivamente amamentadas nos seis primeiros meses justifica o maior peso das meninas e meninos a partir do terceiro e do quarto mês de vida, respectivamente, quando comparados ao padrão.

Nos meninos, o peso médio ao nascer foi semelhante à NCHS/1978, tornando-se maior nas idades subsequentes; nas meninas, mostrou-se maior do nascimento ao sexto mês. De modo geral, as crianças alimentadas com fórmulas têm uma maior ingestão calórica, portanto maior velocidade de crescimento ${ }^{29}$. No entanto, as crianças da referência, alimentadas predominantemente com fórmulas, mostraram um peso médio menor que as do estudo.

Quanto à altura, o comprimento médio das crianças ao nascer foi menor que os resultados assinalados por Jaimovich et al. ${ }^{20}$, mas, do primeiro ao sexto mês, o valor foi semelhante ao apontado por esse estudo. Quanto à pesquisa de Otaigbe et al. ${ }^{6}$, o comprimento médio foi menor ao nascer e no primeiro mês, mas semelhante nas outras idades.

Quanto ao comprimento médio por sexo, verificou-se que nas meninas essa medida manteve-se abaixo do valor encontrado por Donma \& Donma ${ }^{15}$, do nascimento ao sexto mês; nos meninos, ao nascer e no primeiro mês. Ambos os sexos mostraram valores inferiores aos encontrados por Yoneyama et al. ${ }^{17}$, ao nascer, no terceiro mês e no sexto. No entanto, no presente estudo, meninos e meninas obtiveram um aumento no comprimento acima do esperado para o primeiro semestre.

Apesar do comprimento médio das crianças estudadas ter sido menor ou semelhante ao padrão WHO/2006 e à referência NCHS/1978 nas idades avaliadas, elas mostraram crescimento satisfatório e comparável.

Muito embora as crianças do estudo tenham alcançado as expectativas de crescimento, devem-se considerar algumas limitações metodológicas da pesquisa. A mais importante é, sem dúvida, o viés de seleção decorrente da amostra de conveniência, oriunda de um serviço de referência em aleitamento materno, o que impossibi- 
lita uma generalização externa, apesar dos resultados animadores. Outra limitação refere-se à motivação que as mães receberam para participar do estudo e cumprir as recomendações do aleitamento exclusivo, que teve implicações positivas no desfecho do estudo.

\section{O N CLUS Ã O}

O crescimento ponderal das crianças assemelhou-se mais ao modelo do padrão WHO/ 2006 que ao da referência NCHS/1978. Quanto ao crescimento linear, as crianças mostraram um desempenho adequado, comparável ao padrão e à referência nas idades avaliadas.

Os resultados do presente estudo permitem afirmar que a adoção, nos estabelecimentos de saúde, de medidas que favorecem a amamentação é um dos fatores que promovem um ótimo crescimento infantil nos primeiros seis meses de vida. O monitoramento do crescimento infantil deve ser iniciado após o nascimento, de modo longitudinal, por meio de consultas mensais, utilizando-se como referencial de avaliação um padrão cujas curvas sejam instrumentos valiosos para identificar crianças que estão crescendo bem ou em situação de risco.

\section{A GRADECIMENTOS}

Às acadêmicas do curso de Medicina da Universidade Federal do Maranhão, Ana Caroline Alves, Caroline Oliveira, Juliana Cavalcante, Taciana Moura, Thalitta Ferreira; às profissionais de enfermagem Jandira Alves e Jacqueline Cantanhêde; e a todas as mães e crianças que participaram deste estudo.

\section{COLABORADORES}

MGM JALDIN desenvolveu a proposta do estudo para torná-la uma dissertação de mestrado, tendo conduzido todas as etapas do processo, como: elaboração do anteprojeto, construção de questionário, seleção das crianças, verificação das medidas antropométricas, busca nas bases de dados, redação e defesa.
FS PINHEIRO contribuiu nas etapas do processo da dissertação de mestrado e em sua reelaboração sob a forma de artigo, na condição de orientadora da pesquisa. AM SANTOS e NC MUNIZ responsáveis pelo tratamento estatístico dos dados.

\section{REFERÊNCIAS}

1. Marques RFSV, Lopez FA, Braga JAP. O crescimento de crianças alimentadas com leite materno exclusivo nos primeiros 6 meses de vida. J Pediatr. 2004; 80(2):99-105

2. Abiona TC, Onayade AA, ljadunola KT, Abayomi IO, Makanjuola RO. Growth pattern of exclusively breast-fed infants during the first six months of life in lle-Ife, Osun State, Nigeria. Nutr Health. 2002; 16(4):301-12. doi: 10.1177/0260106002016004 04.

3. Donma MM, Donma O. The influence of feeding patterns on head circumference among Turkish infants during the first 6 months of life. Brain Dev. 1997; 19(6):393-7. doi: 10.1016/S0387-7604(97)0 0041-7.

4. Araújo CLP. Avaliação nutricional de crianças. In: Kac G, Sichieri R, Gigante DP. Epidemiologia nutricional. Rio de Janeiro: Atheneu; 2007.

5. Kuczmarski RJ, Ogden CL, Guo SS, GrummerStrawn LM, Flegal KM, Mei Z, et al. 2000 CDC growth charts for the United States: methods and development. Vital Health Stat. 2002; 11(246): 1-189.

6. Otaigbe BE, Alikor EAD, Nkanginieme KEO. Growth pattern of exclusively breastfed in the first six months of life: a study of babies delivered as the University of Port Harcourt Teaching, River State, Nigeria. Niger J Med. 2005; 14(2):137-45.

7. Dewey KG, Peerson JM, Brown KH, Krebs NF, Michaelsen KF, Persson LA. Growth of breast-fed infants deviates from current reference data: a pooled analysis of US, Canadian, and European data sets. World Health Organization Working Group on Infant Growth. Pediatrics. 1995; 93(3): 495-503.

8. Victora CG, Morris SS, Barros FC, Onis M, Yip R. The NCHS reference and the growth of breast and bottle-fed infants. J Nutr. 1998; 128(7):1134-8.

9. Garza C, Onis M. A new international growth reference for young children. Am J Clin Nutr. 1999; 70(1):169-72.

10. World Health Organization. An evaluation of infant growth: the use and interpretation of anthropometry in infants. Bull World Health Organ. 1995; 73(2): 165-74. 
11. Victora CG, Araújo C, Onis M. Uma nova curva de crescimento para o século XXI [acesso 2007 mar 20]. Disponível em: <http://189.28.128.100/ nutricao/docs/geral/nova_curva_cresc_sec_ $x x i . p d f>$.

12. Gökçay G, Turan JM, Partalci A, Neyzi O. Growth of infants during the first year of life according to feeding regimen in the first 4 months. J Trop Pediatr. 2003; 49(1):6-12. doi: 10.1093/tropej/49.1.6.

13. Simoes EAF, Pereira SM. The growth of exclusively breastfeld infants. Ann Trop Paedatr. 1986; 6(1): 17-21.

14. Agostini C, Gianni ML, Silano M, Torcoletti M, Giovannnini M, Riva E. Growth patterns of breast fed and formula fed infants in the 12 months of life: an Italian study. Arch Dis Child. 1999; 81(5): 395-9.

15. Donma MM, Donma O. Infant feeding and growth: a study on Turkish infants from birth to 6 months. Pediatr Int. 1999; 41(5):542-8.

16. Dewey KG, Heinig MJ, Nommsen LA, Peerson JM, Lönnerdal B. Growth of breast-fed and formulafed infants from 0 to 18 months: the darling study. Pediatrics. 1992; 89(6):1035-41.

17. Yoneyama K, Nagata H, Asano H. Growth of Japanese breast-fed and bottle-fed infants from birth to 20 months. Ann Hum Biol. 1994; 21(6): 597-608.

18. Leite AJM. Desenvolvimento infantil. Fortaleza: Ipred; [s.d].

19. Augusto RA, Souza JMP. Crescimento de crianças em aleitamento materno exclusivo no primeiro semestre de vida. Rev Bras Crescimento e Desenvol Hum. 2007; 17(2):1-11.

20. Jaimovich E, Campos C, Hodgson MI, López JM. Lactancia maternal y crecimiento pondoestatural durante el primer año de vida. Rev Chil Pediatr. 1987; 58(3):208-12. doi: 10.4067/S0370-41061 987000300004
21. Sirikulchayanonta C, Boonmee K, Temcharoen P, Srisorrachata S. Birth weight doubling time of Thai infants. Singapore Med J. 2002; 43(7):350-3.

22. Waterlow JC, Thomson AM. Observations on the adequacy of breast-feeding. Lancet. 1979; 2(8136): 238-42.

23. Kumari S, Pruthi PK, Mehra R, Sehgal H. Breastfeeding: physical growth during infancy. Indian J Pediatr. 1985; 52(414):73-7. doi: 10.1007/B F02754723.

24. Sekiyama M, Matsu'ura S, Ohtsuka R. Monthly growth and milk intake of japanese infants exclusively breast-fed up to three months old. Anthropol Sci. 2003; 111(4):373-81. doi: 10.1537/ase. 111.373.

25. World Health Organization. Multicentre growth reference study group. Breastfeeding in the WHO multicentre growth reference study. Acta Pediatr. 2006; Suppl 450:16-26. doi: 10.1080/0803532050 0495423.

26. Duncan B, Schaefer C, Sibley B, Fonseca NM. Reduced growth velocity in exclusively breast-fed infants. Am J Dis Child. 1984; 138(3):309-13.

27. Butte NF, Lopez-Alarcon MG, Garza C. Nutrient adequacy of exclusive breastfeeding for the term infant during the first six months of life. Geneva: WHO; 2002.

28. King FS. Como ajudar as mães a amamentar. Brasília: MS; 1998.

29. Dewey Kg, Heinig Mj, Nommsen La, Lonnerdal B. Low energy intakes and growth velocities of breastfed infants: are there functional consequences? In: Schurch B, Scrimshaw NS. Activity, energy expenditure and energy requirements of infants and children. Lousanne: IDECG; 1990.

Recebido em: 29/9/2011 Versão final em: 19/9/2012 Aprovado em: 2/10/2012 